\title{
Finite Element Analysis of Shaft of Centrifugal Pump
}

\author{
Pramod J. Bachche ${ }^{1}$, R.M.Tayade ${ }^{2}$ \\ ${ }^{1,2}$ (Mechanical Dept., V.J.T.I Mumbai, India)
}

\begin{abstract}
Centrifugal pump is world one of the oldest water pumping devises. The current work deals study Shaft of centrifugal pump for static and dynamic analysis. As we know rotodynamic machineries are designed keenly as there is lot of fluctuation in the loads and speeds. The shaft is analyzed by using finite element analysis technique for stresses and deflections. The total work is carried out in two stages first stage is static analysis. In this stage pump shaft is analyzed for stresses and deflection and same results are verified using graphical integration method. And second for dynamic analysis, in this stage result obtained by static analysis are used to calculate dynamic forces coming in pump shaft. Again shaft is analyzed in dynamic input condition and results are verified by using graphical integration method. The software used for the finite element meshing is HYPERMESH and solver used is RADIOSS. Result values obtained for deflection and stresses are compared in both cases. Result obtained by graphical method and FEA are nearly similar and are in acceptable limits.
\end{abstract}

Keywords: Centrifugal pump shaft, linear static analysis, dynamic analysis, graphical integration method.

Accepted Date: 24 June 2013

\section{Introduction}

Pump is world one of the oldest water pumping devises. Pumps have a place of importance .pumps have now been developed for every possible duty of lifting or raising the pressure of liquids with quantities ,pressure, temperature and viscosity varying within the boundaries and also varying input powers. First type of pump is reciprocating pump . piston pushes the liquid to rise in velocity and pressure. Widely used for developing very high pressure at low flow rate. Another type is rotary pump they obtain their pumping effect by passing their rotating elements through the same space again and again. These are true positive displacement pumps. common examples in this category are gear pump, lobe pump and vane pump etc.

however, the most widely used type of pumps are 1 centrifugal pumps. The use of centrifugal force to lift the liquid from lower level to higher one. Briefly working of centrifugal pump . it comprises of impeller rotating at high speed within the stationary casing or volute. Liquid led into the centre of the impeller is picked up by the impeller vanes and accelerated to high velocity by rotation of impeller and then discharged by centrifugal force into the casing and out to the discharge piping .when the liquid is forced away from the centre a vacuum is created and more liquid flows in.

1.2.problem definition

for given selected shaft the study is carried out for following parameters.

a)static and dynamic deflection of the pump shaft is determined using finite element analysis.

b)stresses developed in pump shaft for static and dynamic condition using finite element analysis.

c) static and dynamic deflection of the pump shaft using graphical integration method.

1.2.1.Shaft specifications

Details if the pump shaft are as follows . these parameters are mainly needed to carry out all the FEA and graphical calculations .

1.overall length $=593 \mathrm{~mm}$,

2. weight of coupling $=12 \mathrm{~kg}$,

3. weight of shaft $=9.5 \mathrm{~kg}$,

4. Weight of impeller $=8.5 \mathrm{~kg}$

5.bearing span $=122.5 \mathrm{~mm}$,

6. working speed $=2900 \mathrm{rpm}$.,

\section{Geometrical Modeling}

Modeling of the shaft is done by using software package PRO-ENGG. WILDFIRE 3.0. modeling is strictly produced considering drawing provided. Small slots and keyways are also considered in the modeling but at time of meshing small entities are suppressed as they cause failure of elements of meshing. Figure below shows cad drawing. 


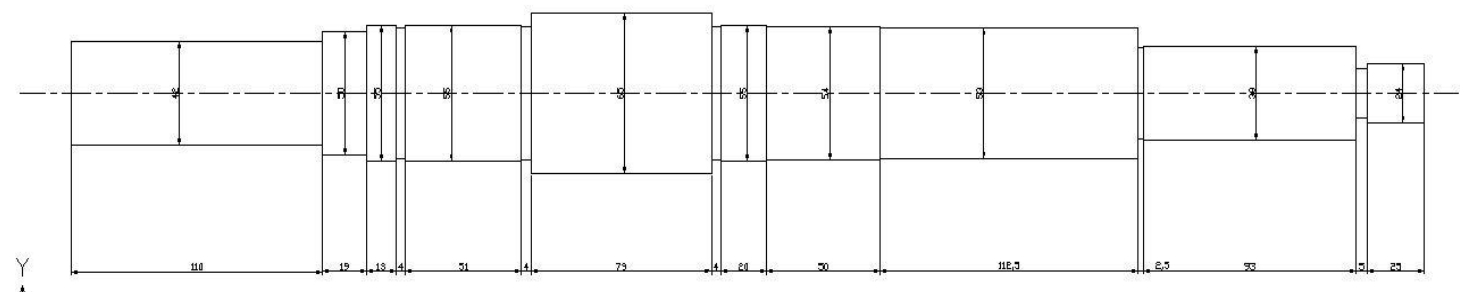

Fig.1 cad drawing of shaft.

III. Discritization or Meshing

2-D thin shell and 1-D beam element supports 6 dofs, but all solid elements have only 3 translational dofs (no rotational dof ) i.e. a 10 nodded tetra element has total of $10 \times 3=30$ dofs.

our shaft Is meshed by using 3D solid elements . elements used are hexahedral and pentahedral elements.

\subsection{Brick or Hex element-}

Brick meshing (also known as Hex meshing) is all about planning, hard work and patience. Brick meshing supports only manual and semi automatic meshing options.

The procedure for brick meshing of complicated parts is to first create a shell mesh on the surfaces and then convert this to hex elements by using the meshing commands extrude, spin, sweep, linear solid,etc. Free face check is very important after the completion of hex meshing.

- Hexahedral elements are solid elements which are extracted from 2D quad elements.

- Hypermesh can generate 8 to 20 nodded hex elements.

- Hex element possesses 3DOF (T T T 000 ) at each node.

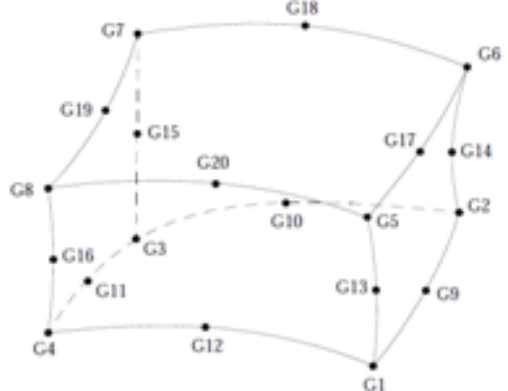

Fig.2. brick element

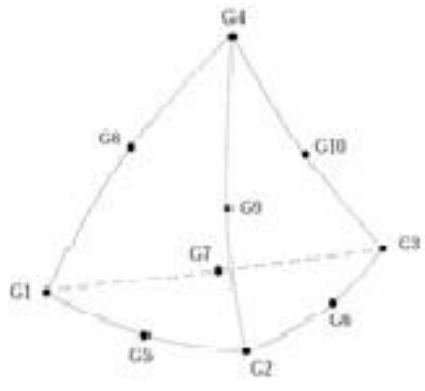

Fig.3.penta element

\subsection{Penta or Wedge element-}

- pentahedral elements are solid elements which are extracted from 2D tria elements.

- Hypermesh can generate 6 to 15 nodded penta elements.

- penta element possesses 3DOF (T T T 000 ) at each node.

By using above mentioned elements modeled shaft will is shown below-

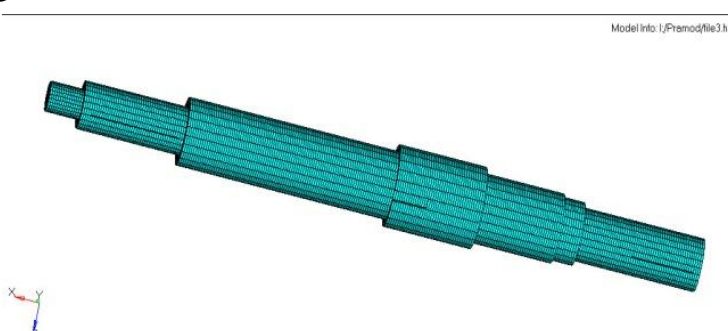

Fig.4. meshed component

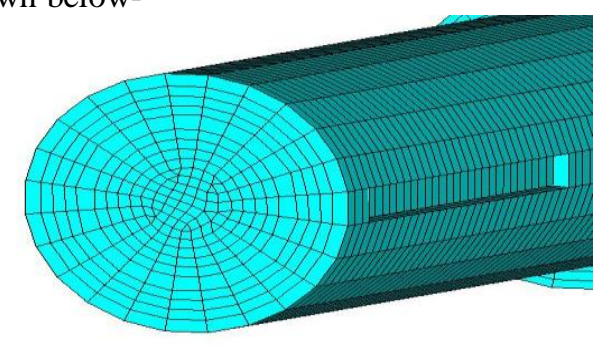

Fig.5. mesh flow 


\begin{tabular}{lccc}
\multicolumn{4}{c}{ IV. Material } \\
• Material name - Carbon Steel IS1570Gr40C-8 \\
Element & $\begin{array}{l}\text { Melt analysis, percent } \\
\text { Min. }\end{array}$ & Max. & Permissible Variation, percent \\
& 0.35 & 0.45 & +0.02 \\
Carbon & 0.10 & 0.35 & +0.03 \\
Silicon & 0.60 & 0.90 & +0.04 \\
Manganese & 0.035 & & +0.005 \\
Sulphur --- & 0.035 & & +0.005 \\
Phosphorus --- & &
\end{tabular}

\section{Linear Static Analysis}

In a linear static analysis displacements, strains, stresses, and reaction forces under the effect of applied loads are calculated.

\subsection{Load application:}

As the shaft is having variable cross section we can not take self weight as single load , due to large self weight it can not be ignored also. We have calculated the weight of each interval . that weight is considered as point load in graphical integration method.

Weight of coupling and weight of impeller is taken as point loads at their position .

- self weight of shaft is considered .

- Weight of impeller and coupling is consider as point load at appropriate locations

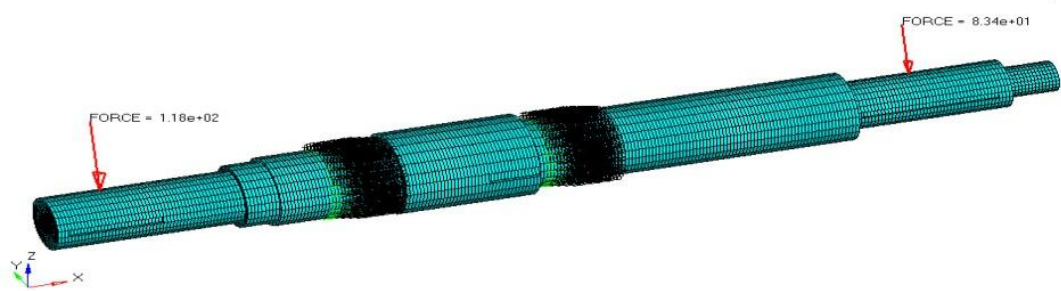

Fig.6.load application

Once all the pre- processing (i.e. meshing, loading and boundary conditions ) the model is then checked. Model is exported with same solver. Small error correction if any is done. Solver and output request are given. Radios is used as solver . then model is solved in same for static analysis.

\subsection{Results}

Once result file is generated then for post-processing HYPERVIEW software is used. All the result available are graphical contour as well as numerical values. static deflection and stresses: the value of deflection is maximum at the impeller end and minimum at bearing locations . there is also considerable deflection at coupling end. following fig [7] gives output image for deflection. max deflection=-4.838 micron .(negative sign indicates downward direction )

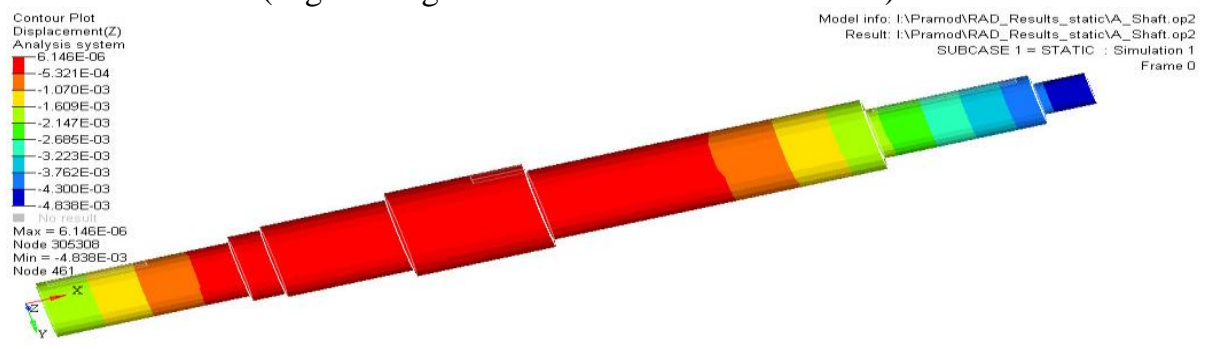

Fig.7.displacement

It is observed that stresses are maximum at the location of bearing .

Max stress $=2.23 \mathrm{~N} / \mathrm{mm} 2$

Following figure shows plotted results of deflection and stresses along y axis and distance along shaft axis on $\mathrm{x}$ axis. 


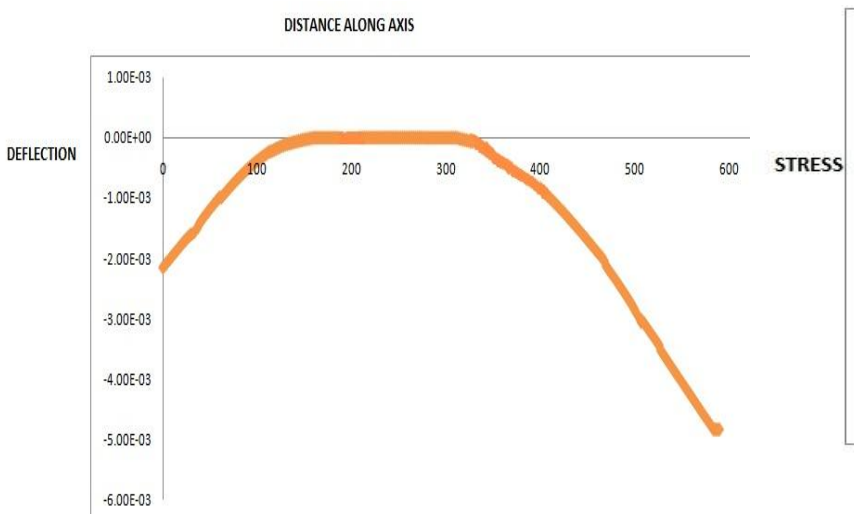

Fig.8.deflection graph

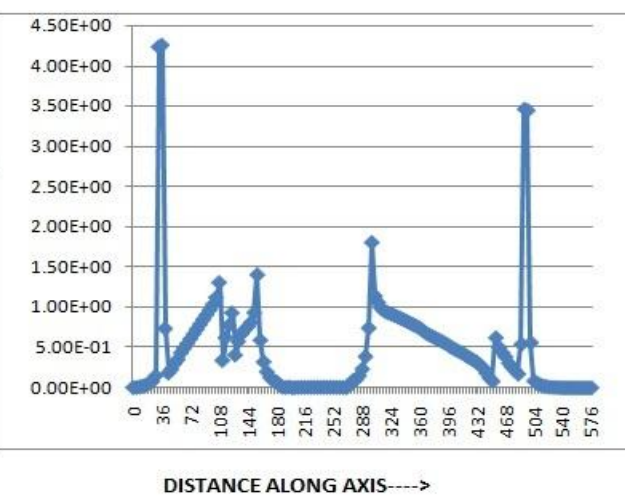

Fig.9.stress graph

\subsection{1..Validation -}

- Method - graphical Integration Method-

above results obtained are cross-verified for deflection. If both the method gives same result then the result obtained are correct. We know that,

$$
\frac{\mathrm{d}^{2} \mathrm{y}}{\mathrm{dx} \mathrm{x}^{2}}=\frac{\mathrm{M}}{\mathrm{EI}}
$$

Where,

M=Bending moment,

$\mathrm{I}=$ Moment of inertia of beam cross section about N.A.,

$\mathrm{E}=$ Young's modulus of elasticity of beam material,

$\mathrm{y}=$ deflection of beam at distance $\mathrm{x}$,

$\mathrm{x}=$ distance from end of beam to the section where the deflection is to be determined.

A straight analytical solution of the equation by double integration is quite tedious for multiple loads and for beams that have changes in cross section ,such as stepped shaft. Due to its advantages such as simplicity and generality graphical integration method is preferred. Firstly M/EI diagram is integrated graphically to get slope diagram . on integration of slope diagram we get deflection diagram.

\subsubsection{Assumptions}

1.shaft is assumed as simply supported beam.

2.shaft is divided into small interval.

3.weight of each interval of shaft is considered as point load acting at centre of that interval.

In graphical Integration Method

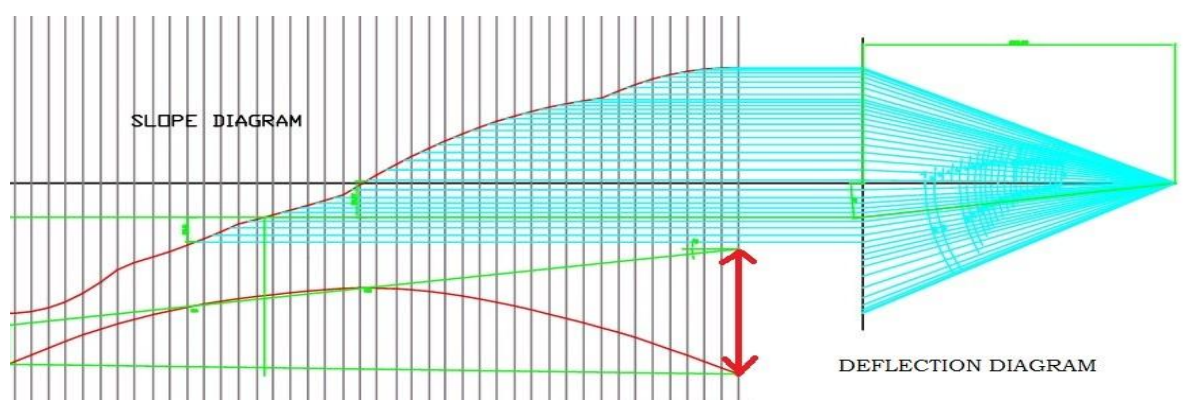

Fig.10. deflection diagram

\subsubsection{Results by Graphical integration method}

- Deflections at support is zero

- $\quad$ Maximum deflection $=4.65 \mathrm{E}-3 \mathrm{~mm}$

- $\quad$ Slope at support $1=7.178 \mathrm{E}-6 \mathrm{rad}$

- $\quad$ Slope at support 2=1.055E-5 rad

- Graphical method is slightly approximate method. Accuracy of result is depends upon no of interval and accuracy of drawing parallel lines.

- $\quad$ Error $=\{(4.838 \mathrm{E}-3-4.65 \mathrm{E}-3) / 4.838 \mathrm{E}-3\} \times 100$

$$
=4.8859 \%
$$




\section{Dynamic Analysis:}

The static deflection produces a displacement of the centre of gravity which, when the shaft rotates ,gives rise to the centrifugal force . these force produces dynamic deflection which is superimposed on the static deflection of shaft. While solving the problem it is assumed that shaft is accurately balanced and hence eccentricity due to imbalance is neglected. Also when the pump is operating large radial thrust acts on the shaft. At minimum flow radial thrust is maximum. As the aim of analysis was to determine shaft deflection under maximum loading, the shaft is analyzed for minimum flow condition.

- By using flow parameters and discharge the radial thrust is calculated by .using V. S. Labanoff [4]

radial thrust $\mathrm{p}=2962.62 \mathrm{~N}$

- Centrifugal force(F) for each intervals average deflection (y)is calculated using formula,

C.F $=\left(\mathrm{W} \omega^{2} \mathrm{y}\right) / \mathrm{g}$

- Then C.F and radial thrust is added .

- $\quad$ Resultant force is applied to midpoint position of each interval.

Following is finite element model of the shaft for dynamic analysis. Though it is called dynamic analysis it is solved in same manner as static analysis. As static deflection is small quantity the load coming on each step is almost same and is equal to radial trust.

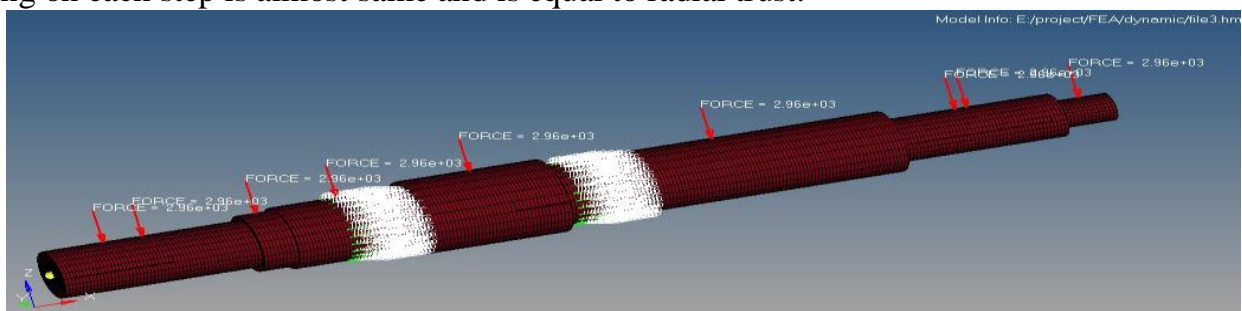

Fig.11. dynamic analysis model

\subsection{Dynamic analysis results:}

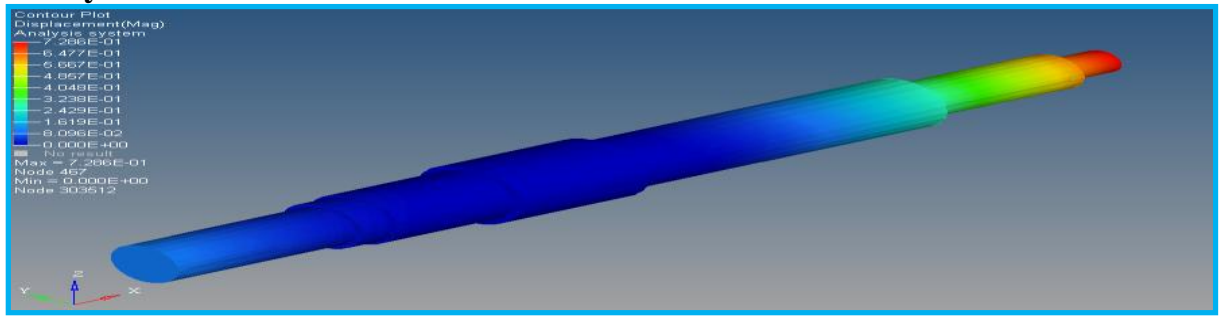

Fig.12. dynamic deflection

Deflections:

maximum deflection $=0.783 \mathrm{~mm}$ at impeller end side.

Deflection at impeller location $=0.212 \mathrm{~mm}$

Deflection at drive end side $=0.103 \mathrm{~mm}$

Stresses :

Maximum stress(VON MISES $)=238 \mathrm{MPa}$

Maximum shear stresses $=132 \mathrm{MPa}$
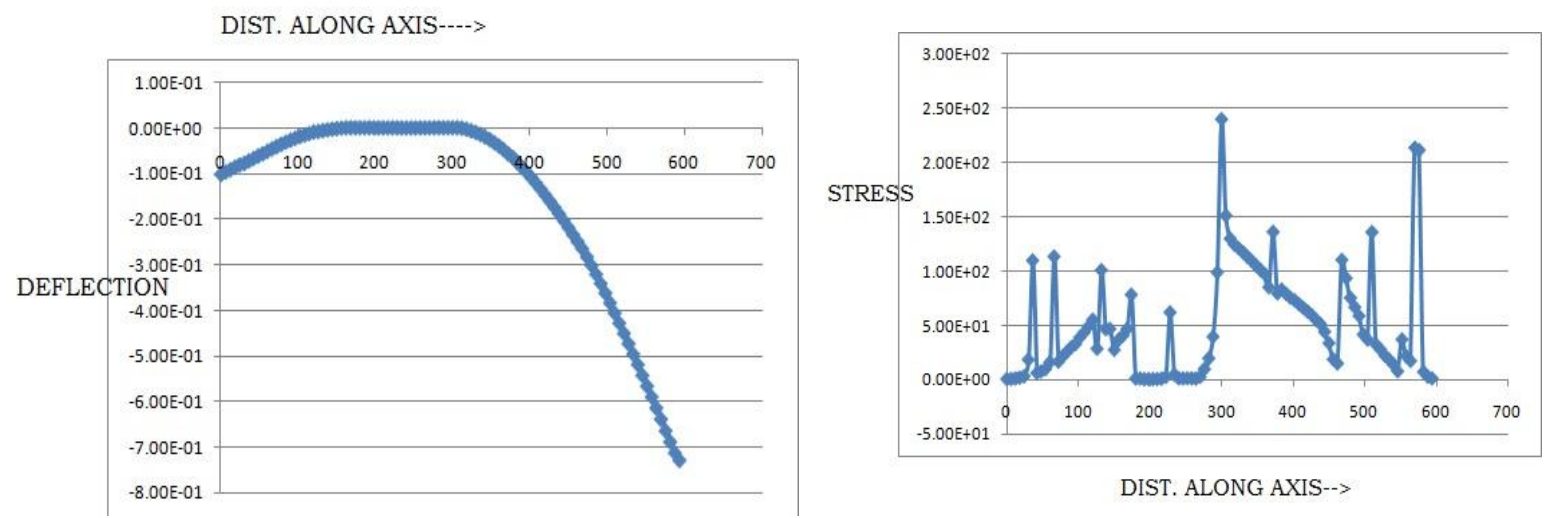

Fig.13 .deflection and stress graphs

\subsection{Validation:}

DIST. ALONG AXIS-->

- Method - graphical Integration Method- all the procedure is same as static analysis . 


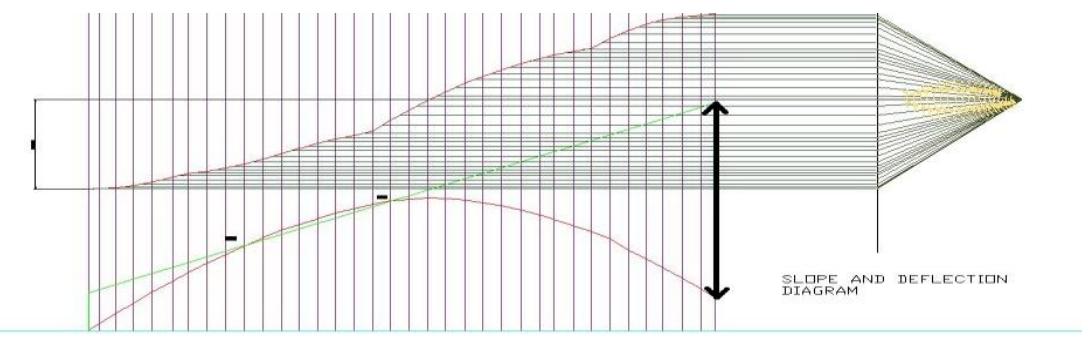

Fig. 14. Slope and Deflection curve.

\subsubsection{Result by Graphical integration method}

- Deflections at support is zero

- $\quad$ Maximum deflection $=0.8298 \mathrm{~mm}$

- $\quad$ Slope at support $1=7.3044 \mathrm{E}-4 \mathrm{rad}$

- $\quad$ Slope at support 2=8.4716E-4 rad

- Graphical method is slightly approximate method. Accuracy of result is depends upon no of interval and accuracy of drawing parallel lines.

- $\quad$ Error $=\{(0.8298-0.783) / 0.8298\} \times 100$

$$
=5.637 \%
$$

\section{Conclusion}

Objective of current study was to analyze shaft behavior in static and dynamic condition for deflection and stresses. Results obtained by graphical integration method and finite element analysis are satisfactory and are in working limit for current set of input conditions. Finite element analysis using Hypermesh and Nastran gives accurate output results . graphical integration method is slightly approximate method so there is little deviation in answers obtained by two methods. Maximum deflection and stresses are generated to minimum flow conditions. Maximum dynamic deflection obtained is $0.783 \mathrm{~mm}$ which is less than limiting value of 0.9 $\mathrm{mm}$ and also maximum stresses for dynamic condition is $238 \mathrm{MPa}$ which is less than allowable tensile strength of $290 \mathrm{MPa}$, and also maximum shear stress is which $132 \mathrm{MPa}$ is also below the allowable shear stress of 190 $\mathrm{MPa}$. So we can conclude whatever design made for is safe and shaft will not fail for current working condition

\section{Theses and papers:}

\section{References:}

[1]. Pump shaft failures- a compendium of case studies by F. Brendt,A van Bennekom

[2]. R.k.turton, ' principles of turbomachinary “,E \& F .N. spon ltd ., London 1984

[3]. Failure analysis of sheared shaft of brine recycle pump by shaleh A. Al- Fozan

[4]. V.S.LOBANOFF ,R.R.ROSS ,'CENTRIFUGAL PUMP: DESIGN and APPLICATION ', 2ND edition ,jaico publishing house ,Bombay ,1995

[5]. Centrifugal pump- basic concept operation, maintenance and troubleshooting-anonymous etc

Weblinks:

[6]. www.sciencedirect.com

[7]. http://www.altairhyperworks.com/Product,7,HyperMesh.aspx

[8]. www.altairuniversity.com/tag/radioss

[9]. www.howstuffworks.com

[10]. www.mcnallyinstitute.com etc. 